\title{
Inhaled drug airflow patterns and particles deposition in the paediatric respiratory tract
}

\author{
ZBIGNIEW TYFA ${ }^{1}$, PAULINA JÓŹWIK ${ }^{1}$, DAMIAN OBIDOWSKI ${ }^{1 *}$, PIOTR REOROWICZ ${ }^{1}$, DANIEL JODKO ${ }^{1}$, \\ KAROLINA KAPKA ${ }^{2}$, RYSZARD MAKOSIEJ ${ }^{2}$, ElŻBIETA CZKWIANIANC ${ }^{2}$, KRZYSZTOF JÓŹWIK ${ }^{1}$ \\ ${ }^{1}$ Institute of Turbomachinery, Lodz University of Technology, Łódź, Poland. \\ ${ }^{2}$ Department of Gastroenterology, Allergology and Pediatrics, \\ Polish Mother's Memorial Hospital Research Institute, Łódź, Poland.
}

\begin{abstract}
Purpose: The effectiveness of inhaled drugs is strictly related to areas reachable by drug particles. Unless particles reach the desired part of the bronchial tree, their influence might not meet the expectations. Consequently, the disease progress might not be stopped or even slowed down. Therefore, the primary objective of this research was to analyze the airflow patterns and particle deposition of a standard inhaled drug using computational fluid dynamics. Methods: The study was devoted to the analysis of the particle diameter influence on their deposition areas within the entire respiratory tract. Two patient-specific respiratory tract models, for 6 and 12-year-old patients, were reconstructed based on the computed tomography examinations. Numerical analyses were carried out as stationary ones with the constant inflow of the particles of various diameters (within the range of 1-50 $\mu \mathrm{m}$ ). It was proven that depending on the particle size, their deposition within the respiratory tract varies significantly. Results: The vast majority of the particles with diameters over $20 \mu \mathrm{m}$ is gathered on the walls of the throat, whereas particles of diameters $5-15 \mu \mathrm{m}$ are accumulated mainly on the trachea walls, leaving the alveoli insufficiently supplied with the drug particles. Conclusions: The inhaled drug size cannot be treated as negligible factor during the drug spraying. An improper distribution of the particles might not inhibit the symptoms of the asthma. Numerical simulations may improve drugs selection and visualize their distribution along the airways, which might accelerate asthma treatment personalization.
\end{abstract}

Key words: patient-specific model, particle tracking, inhaled drug, airflow CFD simulation, paediatric respiratory tract

\section{Introduction}

Aerosol therapy is a technique for inhaled delivery of substances in the form of a suspension of molecules, in which a gas is a dispersing phase, while a liquid or solid substances forming a mist is a dispersed phase. The aerosol therapy is considered to be one of the most effective methods of supplying drug molecules, especially in the case of respiratory diseases. It is a primary method of treatment of bronchial asthma and it plays an important role during cystic fibrosis and primary ciliary dyskinesia (PCD) treatment. Wellconducted treatment of cystic fibrosis, including aerosol therapy, affects the reduction in the incidence of developing complications with the progression of the disease, such as atelectasis, pneumothorax, hemoptysis or pulmonary hypertension. In the case of PCD, the main objective of the treatment is to prevent the formation of bronchiectasis and to maintain the correct lung function as long as possible [13]. Three of the most prominent advantages of the aerosol therapy are as follows:

a) it allows the drug to be delivered directly to the place where the disease process is underway,

b) the onset of action occurs faster than when administered by any other way,

c) it reduces the risk of systemic side effects [4], [15]. For these reasons, the aerosol therapy in children is a widely accepted and preferred treatment, however,

\footnotetext{
* Corresponding author: Damian Obidowski, Institute of Turbomachinery, Lodz University of Technology, ul. Wólczańska 219/223, 90-924, Łódź, Poland. Phone: +48 42 6312388, e-mail: damian.obidowski@p.lodz.pl

Received: January 23rd, 2020

Accepted for publication: April 15th, 2020
} 
it differs from the adults' treatment. It is associated with distinct respiratory anatomy, airflow dynamics and lower pulmonary deposits. It also results from another master respiratory pattern (higher respiratory rate, shallow breath, pulmonary arrhythmia, crying, stop-breath). Unfortunately, information on the correct use of individual inhalers/nebulizers are collected for a group of adult patients or older children. Thus, in clinical practice, the dose is adapted only based on the child's body mass [1], [3], [6]. A lack of good disease control is the cause of exacerbations, which requires modification of the treatment. A lack of proper control of asthma affects the reduction of daytime activity, reduces exercise capacity and lowers the quality of life of the patient and his family [9].

In the pediatric population, the change in the anatomical and physiological conditions occurring with the age of the child must be taken into account [7], [10], [11]. Hence, the geometry of the airways segment, in which the drug particles are deposited, is crucial for the effects of the treatment. Unfortunately, the proportion of inhaled drugs reaching the lungs to achieve maximum therapeutic efficacy is still very low in children. After inhaled administration, a large part of the dose does not reach the alveoli due to the particle deposition in the oral and throat cavity [23], [24].

Several papers estimate the flow conditions influence on the drug particle deposition areas with the computational fluid dynamics (CFD) tools. However, the authors of those publications based their conclusions on the patient-specific respiratory tract geometries of the adult patients. For instance, Rahimi-Gorji et al. [21] investigated cases of the flow with three different volume flow rates $\left(250,500\right.$ and $\left.1000 \mathrm{~cm}^{3} / \mathrm{s}\right)$ and with the particle size equal to 1,5 and $10 \mu \mathrm{m}$. Luo and Liu [17] based on a slightly more advanced respiratory tract model, but they imposed different volume flow rates $\left(210-630 \mathrm{~cm}^{3} / \mathrm{s}\right)$ and used different sizes of the particles $(2,4,6$ and $10 \mu \mathrm{m})$. The study of Tena and Clarà [25] was focused on the vast theoretical description of numerous factors that affect the deposition of aerosolized drugs. Moreover, it presented results of the CFD analyses of the particle flow (particle size equal to $1 \mathrm{pm}, 5 \mu \mathrm{m}, 50 \mu \mathrm{m}$ ) with a broad range of inlet boundary conditions, i.e., $100-1583 \mathrm{~cm}^{3} / \mathrm{s}$.

Despite providing a very valuable and extensive source of information, the results obtained in the aforementioned papers cannot be taken for granted when it comes to the particle flow analysis for the pediatric patients. Due to their different anatomical characteristics with significantly smaller upper tract geometries, the inhaled drug particles might behave differently. Thus, the deposition areas and/or deposition rates might vary between adults and children.

There is no accurate data concerning factors affecting the deposition in a particular patient in the pediatric population. For this reason, one can find a very limited number of articles covering the aspect of the inhaled drug investigations in the children's respiratory tract. In one of such papers, the authors analyzed the aerosol flow in the anatomically idealized reconstruction of the upper respiratory tracts depending on patients' age [5].

The paramount objective of our research was to conduct numerical simulations of the particle flow to analyze their distribution in the patient-specific upper respiratory tract models of two pediatric patients. The presented research aimed at verifying whether the size of particles influences their deposition on the model walls. Furthermore, it was analyzed whether the assumed ranges of the sizes for each deposition mechanisms can be treated as correct. Moreover, it was assessed whether the obtained dependence (deposition area vs. particle size) resembles the characteristics obtained for adult patients.

\section{Materials and methods}

The models of the upper respiratory tracts were reconstructed basing on computer tomography (CT) data of the 6-year old and 12-year old patients who were diagnosed during standard clinical evaluation. In both cases, the obtained DICOM (Digital Imaging and Communications in Medicine) image sequences started at the middle part of the trachea. Each CT scan, deriving from Philips Brilliance iCT 128 slice scanner with the resolution equal to $0.3789,0.3789$ and $1.0 \mathrm{~mm}$, was loaded into the Mimics Research 20.0 software. After several semi-automatic segmentation techniques and manual corrections, 3D surface models of the upper respiratory tracts were extracted from binary masks. The models were exported in the stereolithography (STL) format and represented the surface models of the patient-specific respiratory tracts. Bearing in mind the observation that the particles of larger diameter tend to deposit on the upper part of the respiratory tract (mouth, throat and trachea), the idealized artificial upper part of the trachea and throat were added to the generated models. For that purpose, a swept blend operation was used - its thorough description is outlined in former publications of the authors [12], [22], [26]. The simplified workflow chart of the model preparation is depicted in Fig. 1, while the final geome- 

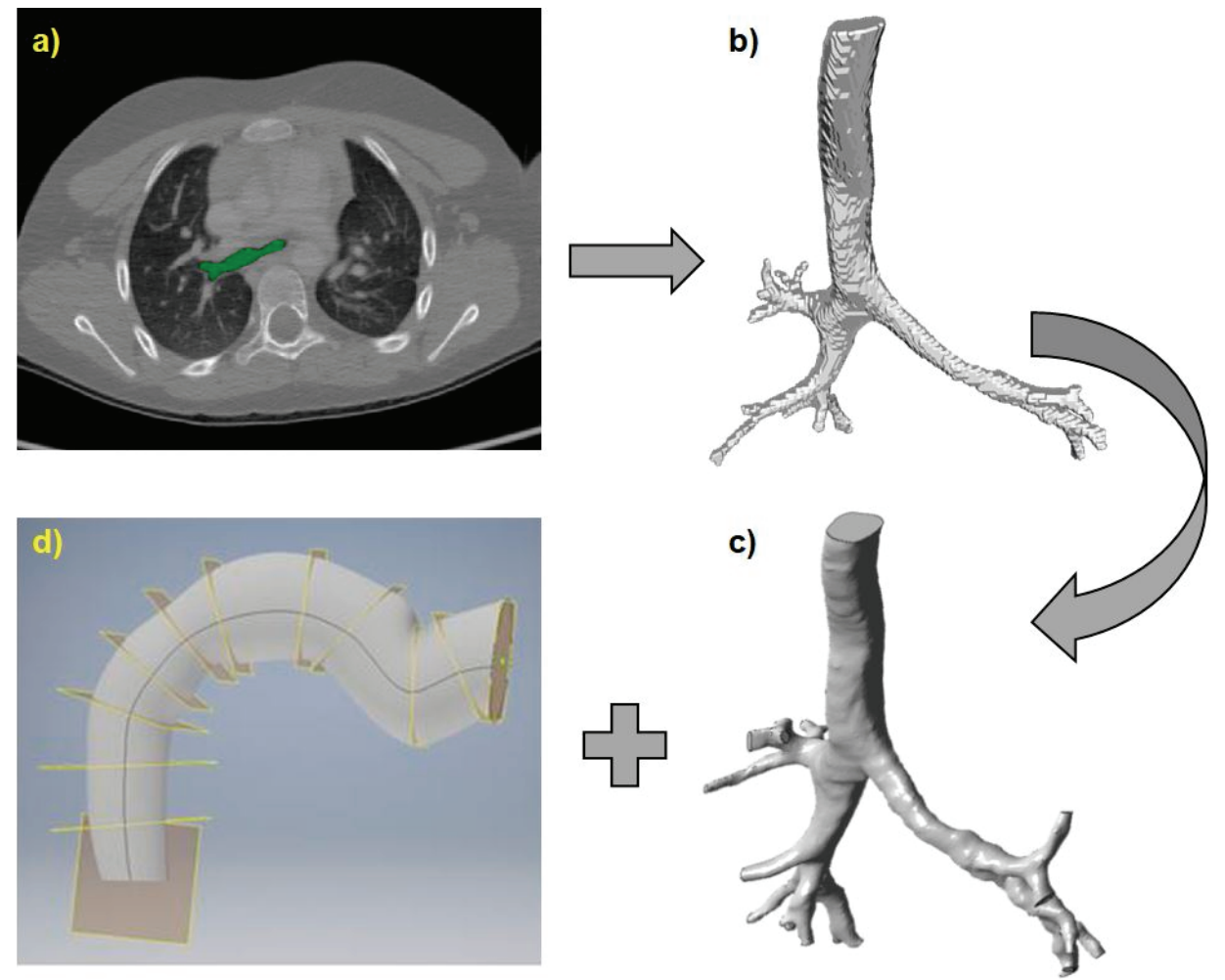

Fig. 1. Simplified workflow chart of the respiratory tract reconstruction process

tries with the artificial trachea and throat are presented in Fig. 2.
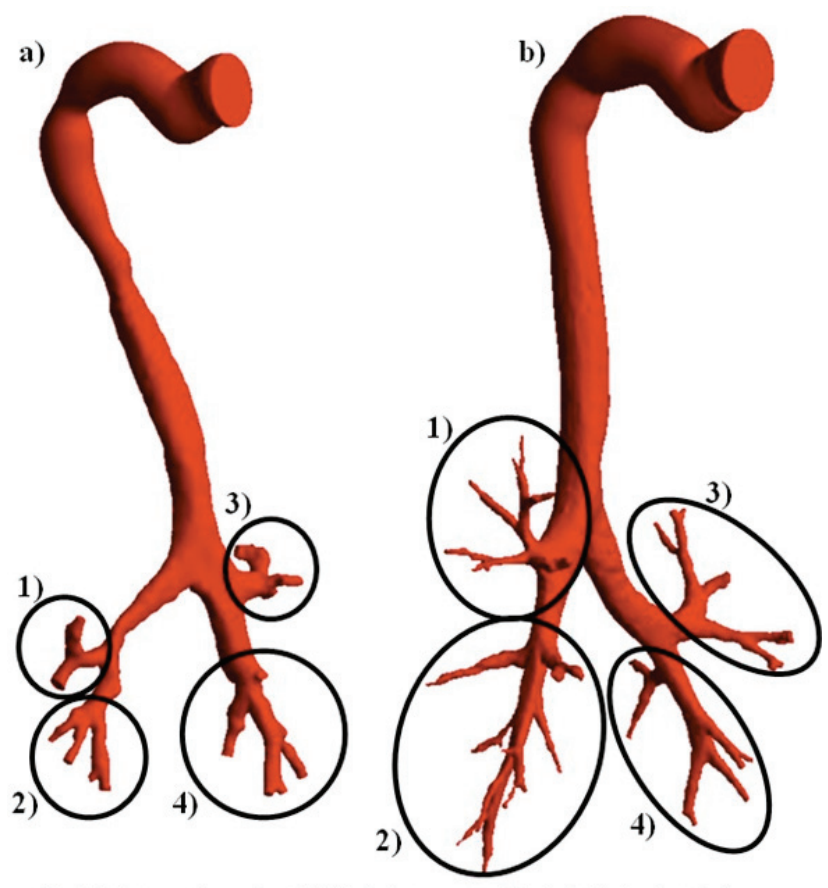

1) right superior $\&$ middle lobes 3) left superior lobe
2) right interior lobe 4) left interior lobe

Fig. 2. Reconstructed models of the respiratory tracts with marked regions of investigations: a) 6-year old patient, b) 12-year old patient

Afterwards, the surface models were imported into the Ansys ICEM software, where volume meshes were prepared. To prevent obtaining too small number of elements in the narrow branches, local refinement of the mesh was introduced. Furthermore, the inflation layer was embedded in mesh domains to obtain as precise results near the model wall as possible. To verify the influence of the mesh density on the calculated values, the mesh independence test was performed, and the most optimal mesh density was chosen. The final meshes (Fig. 3) comprised circa 4.3 million and 7.4 million elements for the 6-year old and 12-year old patient geometries, respectively.

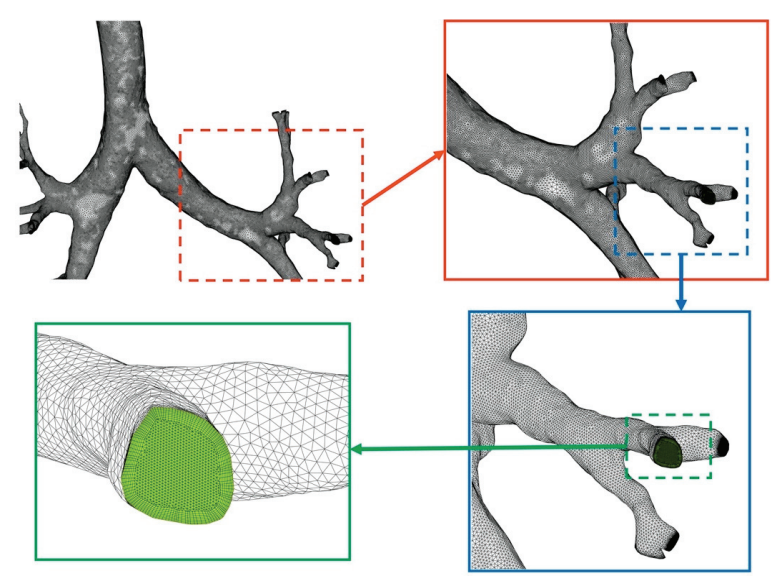

Fig. 3. Numerical mesh for the 12-year old patient geometry 
For the numerical simulations, which were conducted with the use of the Ansys CFX v.18.2 software, the air was defined as incompressible fluid with the constant density, dynamic viscosity and temperature, equal to $1.1644 \mathrm{~kg} / \mathrm{m}^{3}, 1.87 \mathrm{E}-5 \mathrm{~Pa} \cdot \mathrm{s}$ and $303 \mathrm{~K}$ $\left(30{ }^{\circ} \mathrm{C}\right)$, respectively. The authors assumed rigid walls of the respiratory tract models since the data concerning the expandability of those structures for the given patients were unavailable. The drug particles were modeled as spherical-shaped solid structures of constant diameters (within the range of 1-50 $\mu \mathrm{m}$ ) and constant density (i.e., 142, 189 and $236 \mathrm{~kg} / \mathrm{m}^{3}$ ). Densities were assessed experimentally by spraying the inhaled drug on Petri dishes and providing information about the measured mass and volume of the prepared drug powder. In total, 45 cases were investigated for each patient's model. Such a broad spectrum of particle sizes enabled verifying the hypothesis of the correlation between the deposition mechanisms and the particle size. Moreover, the main idea behind such a vast variation of the particle size is the fact that despite the production of the drug with the constant size, the drug particles might combine to the larger aggregates.

The numerical simulations were carried out as stationary ones. At the inlets to numerical domains, the uniform velocity was assumed, equal to $1.0 \mathrm{~m} / \mathrm{s}$. For each investigated case, the drug dose was constant: $40 \mu \mathrm{g}$. The static gauge pressure was set on all the outlet surfaces, which were defined as the "opening" boundary conditions. Relative pressure values for both of the lungs were assumed, i.e., at all the outlets for the 6-year old patient they were equal to $-0.12 \mathrm{kPa}$, whereas for the 12-year old patient they were equal to $-0.14 \mathrm{kPa}$.

Flow solutions were obtained by solving Reynolds-averaged Navier-Stokes equations, which aim at decomposing the instantaneous field variables into time-averaged and fluctuating parts (Eqs. (1) and (2)).

$$
\begin{gathered}
\rho \cdot \frac{\partial U_{i}}{\partial t}+\rho \cdot \frac{\partial}{\partial x_{j}}\left(U_{i} \cdot U_{j}\right)= \\
-\rho \cdot \frac{\partial P}{\partial x_{i}}+\rho \cdot \frac{\partial}{\partial x_{j}}\left(2 \cdot \mu \cdot S_{i j}-\rho \cdot \overline{u_{i}^{\prime} \cdot u_{j}^{\prime}}\right)
\end{gathered}
$$

where $U_{i} U_{j}$ is mean part of velocity, $\overline{u_{i}^{\prime} u_{j}^{\prime}}$ is the fluctuating part of velocity, $P$ is the pressure field, while $S_{i j}$ is the mean strain-rate tensor, expressed by:

$$
S_{i j}=\frac{1}{2} \cdot\left(\frac{\partial U_{i}}{\partial x_{j}}+\frac{\partial U_{j}}{\partial x_{i}}\right) .
$$

In order to solve the three unknown quantities obtained after the aforementioned decomposition, a turbulence model had to be added. For that purpose, the $k$ - $\omega$ Shear Stress Transport (SST) turbulence model was used. It is claimed that it provides sufficient turbulence approximations both in the boundary layer and in the free flow regime [18].

Considering the particle transport theory, particles are tracked through the calculated flow in a Lagrangian way. As far as CFX solver is concerned, Lagrangian tracking integrates particle paths through the numerical domain until their flow is finished. Such a situation occurs either when a particle leaves the domain or when a predefined integration limit is reached. Modeled particles generate source terms to the energy, momentum and fluid mass equations, which form a set of differential equations that are integrated during the computation process. According to the CFX theory guide [2], the particle acceleration is affected by the forces which occur due to two main phenomena: a) the difference in velocity between the particle and fluid; $b$ ) displacement of fluid by the particle. The general equation of the particle motion is shown in Eq. (3).

$$
m_{P} \cdot \frac{d \bar{U}_{P}}{d t}=\bar{F}_{D}+\bar{F}_{B}+\bar{F}_{R}+\bar{F}_{V M}+\bar{F}_{P}+\bar{F}_{B A},
$$

where $F_{D}$ is the drag force acting on the particle, $F_{B}$ is buoyancy with gravitational force, $F_{R}$ are forces due to domain rotation, $F_{V M}$ is the virtual mass force, $F_{P}$ is the pressure gradient force and $F_{B A}$ is the Basset force (history term) [2].

Due to the fact that only a few of the above-mentioned terms have a major influence on the particle transport, some of the forces are neglected during the computation process, for instance, the Basset force. The buoyancy force together with the drag force (which are of major influence) are described below. The buoyancy force is governed by the following formula:

$$
\bar{F}_{B}=\frac{\pi}{6} \cdot d_{P}^{3} \cdot\left(\rho_{P}-\rho_{F}\right) \cdot \bar{g},
$$

where $\rho_{P}$ and $\rho_{F}$ are particle and fluid densities (correspondingly), $d_{P}$ is particle diameter and $\bar{g}$ is the gravity vector.

As far as the drag force is concerned, the governing equation is as follows:

$$
\bar{F}_{D}=\frac{1}{2} \cdot C_{D} \cdot \rho_{F} \cdot A_{F} \cdot\left|\bar{U}_{F}-\bar{U}_{P}\right| \cdot\left(\bar{U}_{F}-\bar{U}_{P}\right),
$$

where $\bar{U}_{F}$ and $\bar{U}_{P}$ are fluid and particle velocities (correspondingly), $A_{F}$ is the effective particle cross section and $C_{D}$ is the drag coefficient. 
The drag coefficient is introduced to account for the experimental results on the viscous drag of a solid sphere and can be calculated basing on the non-linear function of the particle Reynolds number, $R e_{P}$. Such dependency is described below [2].

$$
C_{D}=\left\{\begin{array}{lll}
\frac{24}{R e_{P}} ; & \text { for } & R e_{P}<<1 \\
\frac{24}{R e_{P}} \cdot\left(1+0.15 \cdot R e_{P}^{0.687}\right) ; & \text { for } \quad 0.1<R e_{P}<1000 \\
0.44 ; & \text { for } \quad \operatorname{Re}_{P} \geq 1000
\end{array}\right.
$$

It was assumed that particle influence on the fluid flow as well as the interaction between them, i.e., particle-particle collisions, can be treated as negligible factors, hence, the one-way coupling between the air and particle flow fields was set.

The particle-wall interaction was controlled by the equation dependent method. It means that particle momentum change was governed by two parameters of restitution, i.e., perpendicular and parallel coefficients. The former one was responsible for the normal changes in the momentum, whereas the latter - for the tangential changes. The authors assumed a direct and immediate adhesion of particles to the model wall. When any particle hits the wall, it sticks to its surface and no additional particle sliding motion is calculated. Such an assumption can be justified since walls of the respiratory tract are not completely dry, but are covered with a thin layer of moist epithelial cells. The viscous forces acting on the particle are greater than forces deriving from the particle momentum (where particle mass is extremely small). Thus, when the particle collides with the wall, it immediately sticks to the surface, regardless of normal and tangential velocity values.

\section{Results}

A total deposition of particles on the respiratory tract walls was the first analyzed parameter. Thanks to such an analysis, the authors could easily observe the dependence of the adhered particles locations on their size. Figure 4 depicts the comparison of the particle deposition on the model walls, for both investigated patients. The particle density chosen for this comparison was equal to $189 \mathrm{~kg} / \mathrm{m}^{3}$, whereas the diameters were as follows: 1,15 and $50 \mu \mathrm{m}$. 12-year old patient

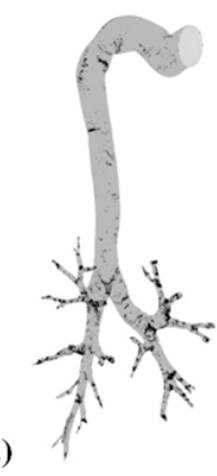

d)

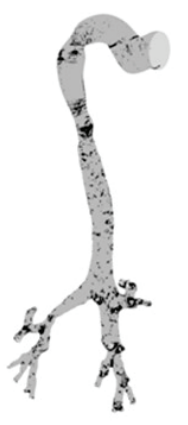

b)

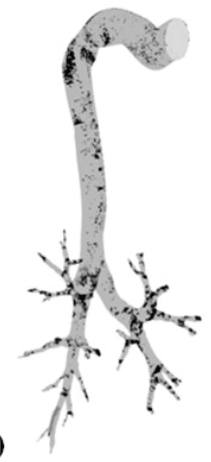

6-year old patient

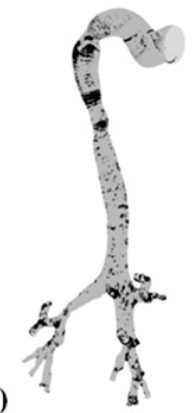

f)

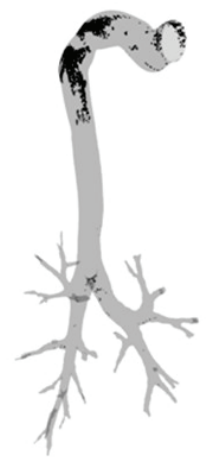

c)

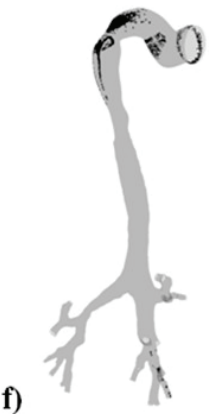

Fig. 4. Particle deposition on the analyzed models - different particle sizes analyzed: a) $1 \mu \mathrm{m}$, b) $15 \mu \mathrm{m}$, c) $50 \mu \mathrm{m}$, d) $1 \mu \mathrm{m}$, e) $15 \mu \mathrm{m}$, f) $50 \mu \mathrm{m}$

Analyzing distributions presented in Fig. 4 one can notice that the particles of the highest diameters tend to settle on the throat and the upper trachea segments. As a result, an insufficient number of drug particles might reach the farther target area. It was proven that when larger particles are concerned, inertia is the main principle of their deposition mechanism. Focusing on the middle-sized particles $(15 \mu \mathrm{m})$, one can observe that they deposited at almost every part of the respiratory tract, even in the vicinity of the second/third generation of bronchi. The distribution of the smallest particles $(1 \mu \mathrm{m})$ seems to be relatively similar to the former case, but with a slightly lower concentration of the particles on the throat and upper trachea walls. Graphical representation does not take into account the particles that leave the numerical domain. For instance, the particles shown in Fig. $4 d$ constitute merely $11 \%$ of the total number of particles, whereas the particles of size $15 \mu \mathrm{m}$ (Fig. 4e) constitute ca. $40 \%$ of the total inflow.

Therefore, to present the data comprising all the obtained values with higher clarity, the results are plotted in the form of the bar charts. Figures 5 and 6 show that for each patient and each density, a sigmoid function describes the particle deposition dependency 


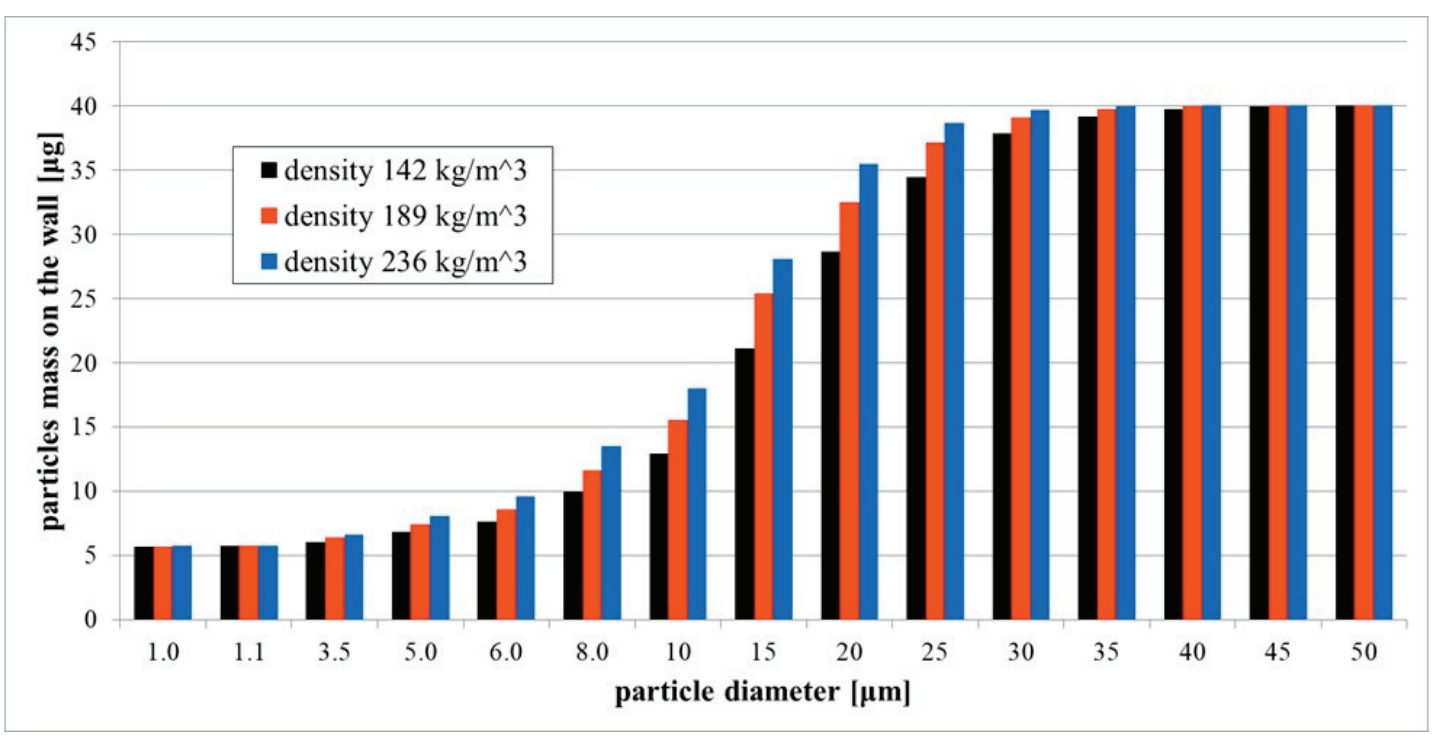

Fig. 5. Total deposition of the particles on the respiratory tract wall for the 12 -year old patient

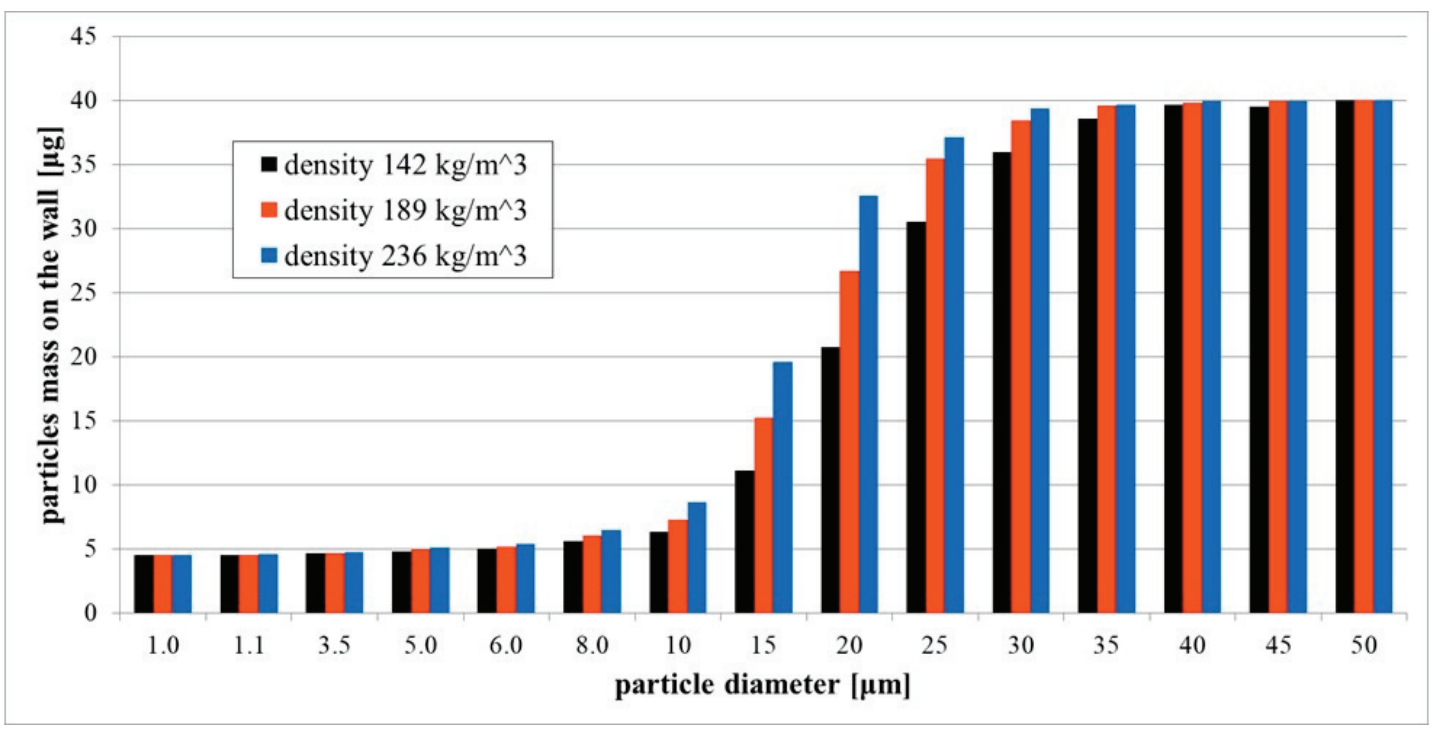

Fig. 6. Total deposition of the particles on the respiratory tract wall for the 6-year old patient

on the particle size. One can observe very similar characteristics for both patients. When the particle size decreases, the overall mass of the deposited particles tends asymptotically towards the value of circa $5 \mu \mathrm{g}$ which is approximately $10 \%$ of the total inflow. When the diameter of the particles exceeds the value of $30 \mu \mathrm{m}$, almost the entire drug dose, i.e., $40 \mu \mathrm{g}$, settles on the respiratory tract walls. The highest gradients between each subsequent particle size occur in the range 6-25 $\mu \mathrm{m}$ and $10-25 \mu \mathrm{m}$ for the 12-year old and 6 -year old patients, respectively. Moreover, it is worth mentioning that with an increase in particle density, those differences tend to increase as well. The normalized comparison of the obtained results is presented in the tabularized form (Table 1).
To assess the impact of the model geometry on the particle deposition, it was decided to compare the cases for both patients on a single plot (Fig. 7). Hence, one could verify whether the particle deposition dependence on their size behaves similarly for the different geometries. The sigmoid characteristics of the obtained data are visible for both patients, although, one can observe higher mass of the deposited particles on the model wall for the 12year old patient. The clearest differences can be noted in the particle diameter range 6-20 $\mu \mathrm{m}$, especially for the case with $15 \mu \mathrm{m}$ particles - the difference between the results for both patients exceeded $10 \mu \mathrm{g}$ (ca. $25 \%$ ). When the smallest or largest particles are concerned, the differences between both 
Table 1. Percentage of the particles deposited on the respiratory tract walls for all the densities and for both patients

\begin{tabular}{|c|c|c|c|c|c|c|}
\hline \multirow{3}{*}{$\begin{array}{c}\text { Particle } \\
\text { size }[\mu \mathrm{m}]\end{array}$} & \multicolumn{6}{|c|}{ Particles deposited on the model wall [\%] } \\
\hline & \multicolumn{3}{|c|}{ 6-year old patient } & \multicolumn{3}{|c|}{ 12-year old patient } \\
\hline & $142 \mathrm{~kg} / \mathrm{m}^{3}$ & $189 \mathrm{~kg} / \mathrm{m}^{3}$ & $236 \mathrm{~kg} / \mathrm{m}^{3}$ & $142 \mathrm{~kg} / \mathrm{m}^{3}$ & $189 \mathrm{~kg} / \mathrm{m}^{3}$ & $236 \mathrm{~kg} / \mathrm{m}^{3}$ \\
\hline 1.0 & 11.3 & 11.3 & 11.3 & 14.3 & 14.3 & 14.4 \\
\hline 1.1 & 11.3 & 11.3 & 11.4 & 14.3 & 14.3 & 14.4 \\
\hline 3.5 & 11.8 & 11.9 & 11.9 & 15.1 & 15.9 & 16.5 \\
\hline 5.0 & 12.0 & 12.3 & 12.6 & 17.0 & 18.5 & 20.2 \\
\hline 6.0 & 12.5 & 12.8 & 13.5 & 19.0 & 21.5 & 24.0 \\
\hline 8.0 & 13.9 & 15.1 & 16.1 & 24.9 & 29.0 & 33.7 \\
\hline 10.0 & 15.8 & 18.2 & 21.6 & 32.3 & 38.8 & 45.0 \\
\hline 15.0 & 27.9 & 38.0 & 48.9 & 52.9 & 63.5 & 70.2 \\
\hline 20.0 & 51.8 & 66.6 & 81.4 & 71.7 & 81.3 & 88.6 \\
\hline 25.0 & 76.4 & 88.6 & 92.8 & 86.0 & 92.8 & 96.7 \\
\hline 30.0 & 89.8 & 96.1 & 98.4 & 94.7 & 97.8 & 99.3 \\
\hline 35.0 & 96.4 & 99.0 & 99.2 & 97.9 & 99.4 & 99.9 \\
\hline 40.0 & 99.1 & 99.5 & 99.9 & 99.3 & 99.9 & 100.0 \\
\hline 45.0 & 98.8 & 100.0 & 100.0 & 99.9 & 100.0 & 100.0 \\
\hline 50.0 & 100.0 & 100.0 & 100.0 & 100.0 & 100.0 & 100.0 \\
\hline
\end{tabular}

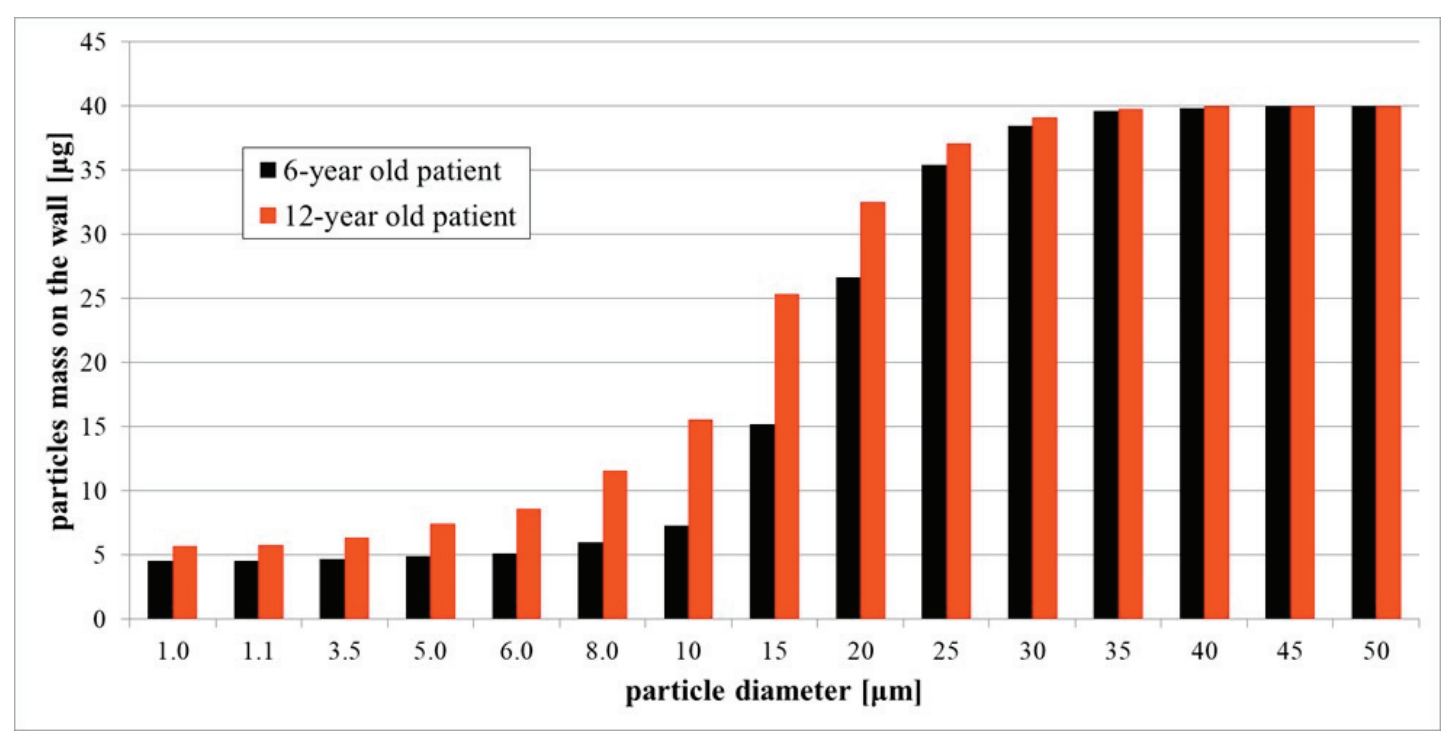

Fig. 7. Total deposition of the particles on the respiratory tract wall for both patients; particle density equal to $189 \mathrm{~kg} / \mathrm{m}^{3}$

cases are not significant and they occur due to the greater area for possible deposition. Thus, this indicates that the model geometry cannot be left unmentioned when defining the proper drug dosage and inhalation technique.

The total deposition of the particles on the respiratory tract wall might give an insight into the basic mechanisms leading to the particle settling. However, from the clinical point of view, the most important aspect is to assess whether the particles reach the desired respiratory structures, e.g., specific part of the lung. Hence, the authors decided to categorize the selected areas (Fig. 2). However, even though the right lung comprises three separate lobes, only 2 separate regions for the analysis were established. The reason for that simplification is connected to the limitations of the reconstructed models, due to CT resolution limits. Furthermore, the authors introduced the variable called particle efficacy $(P E)$ which is described by Eq. (7). The sole deposition particles on the respiratory tract wall does not include the particles that leave the numerical domain, which undoubtedly reach farther bronchi generations and have a better therapeutic effect. Thus, in our opinion, $P E$ is a better illustration of drug delivery than deposition on the walls. In Figs. 8 and 9, the $P E$ dependencies on the particle size for both pediatric patients, are outlined. 
$P E=\frac{\text { number of particles that left the structure }}{\text { total number of particles entering the domain }} \cdot 100 \%$

Focusing on the $P E$ parameter, which defines the particles that left the numerical domain maximizing their chances to reach the alveoli, one can observe the similarity of the functions describing it for both pediatric patients. Generally, a decreasing trend can be observed, especially for the middle-sized and large particles $(>15 \mu \mathrm{m})$. The $P E$ parameter for the particles smaller than $5 \mu \mathrm{m}$ tends to be relatively constant. Nonetheless, all the plots outlining the $P E$ parameter (Figs. 8 and 9) indicate that the smaller the particles, the higher their possible efficacy. However, the particle size decrease tends asymptotically towards a specific, constant value. This means that despite inhaling significantly smaller particles, the therapy efficacy might not be improved. Moreover, one of the available researches indicates that too small particles, i.e.,
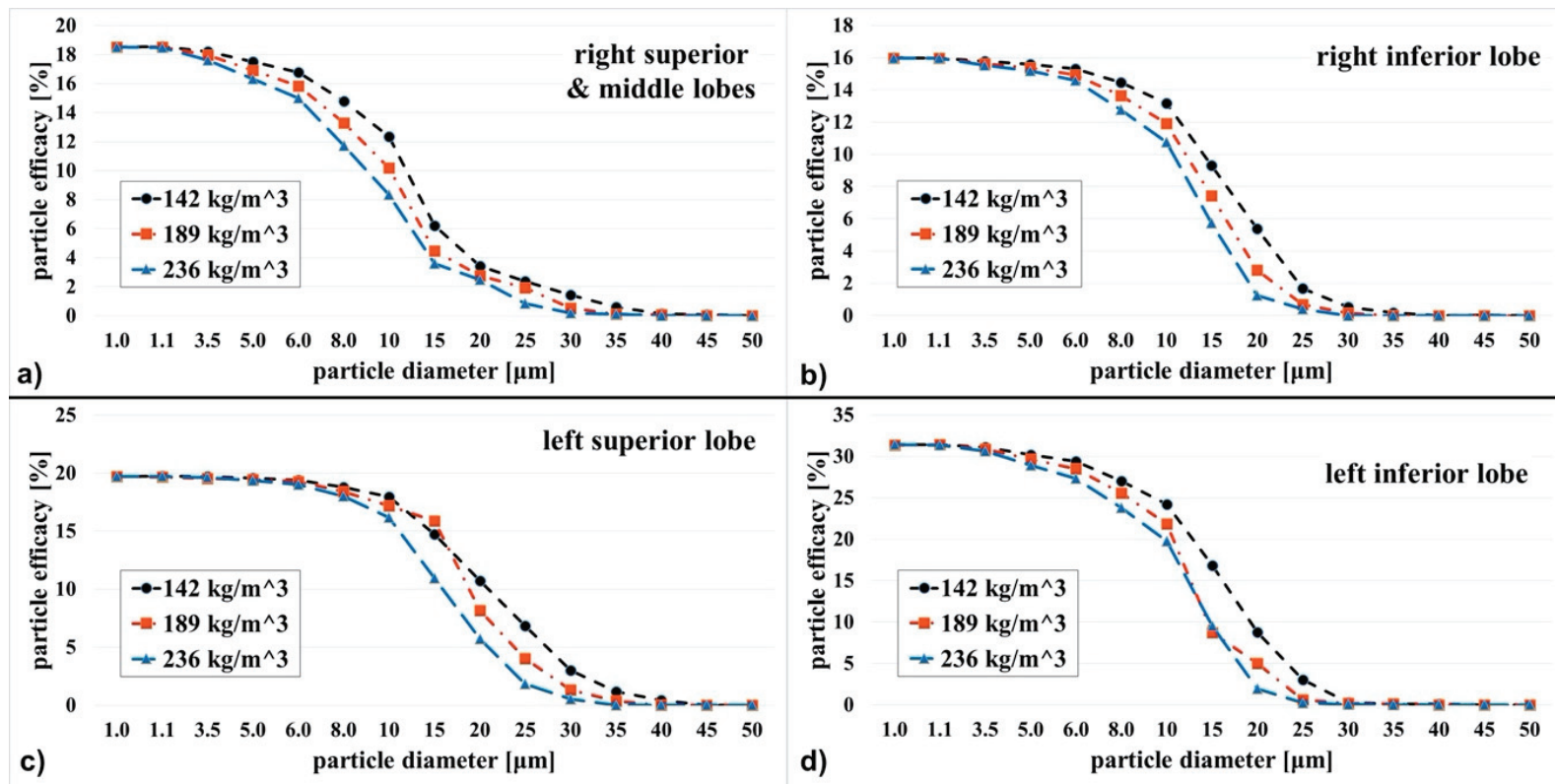

Fig. 8. Deposition factor and particle efficacy dependence on particle size for 12-year old patient's geometry: a) right superior lobe, b) right inferior lobe, c) left superior lobe, d) left inferior lobe
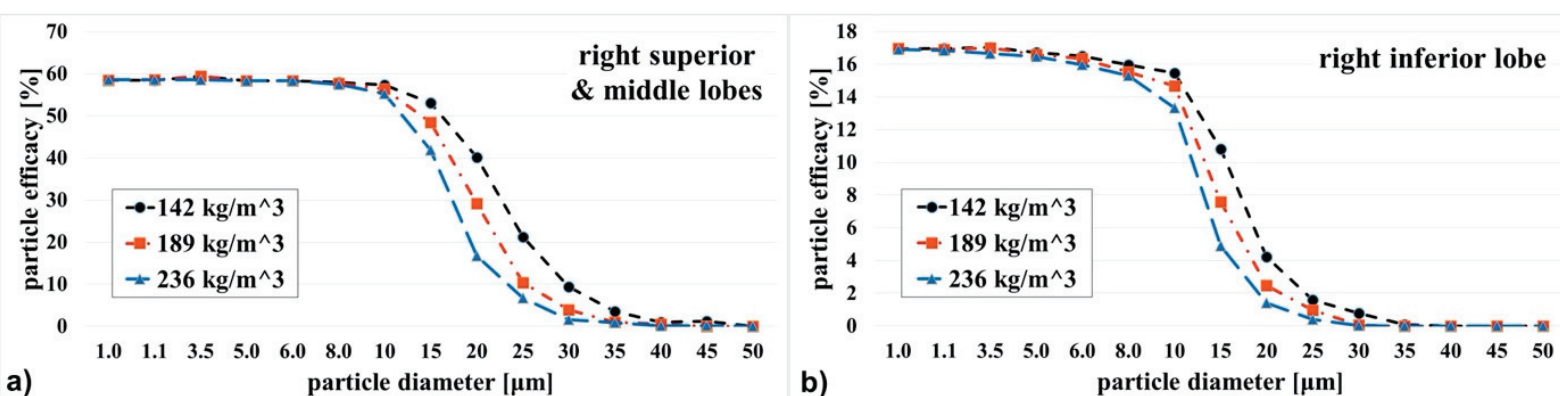

particle diameter $[\mu \mathrm{m}]$

b)
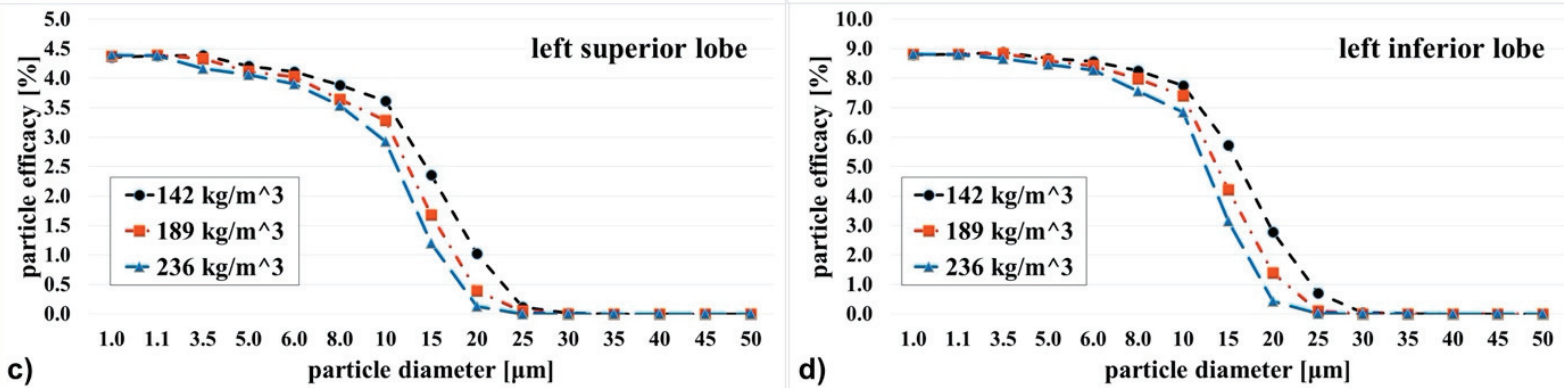

Fig. 9. Deposition factor and particle efficacy dependence on particle size for 6-year old patient's geometry: a) right superior lobe, b) right inferior lobe, c) left superior lobe, d) left inferior lobe 
$<1.0 \mu \mathrm{m}$, might remain in suspension and as a result can be exhaled out of the organism leading to unsuccessful treatment [19].

The $P E$ parameter values obtained for the smaller particles are similar for each analyzed density, however, when their size exceeds ca. $20 \mu \mathrm{m}$, particles of higher density tend to present worse efficacy. This phenomenon is strictly related to higher momentum and inertia of larger particles, which start to deposit on earlier parts of the model walls.

From the numerical analyses performed, the following conclusions can be drawn.

The smallest particles tend to flow out of the numerical domain since their inertia does not cause them to impact the model wall (their flow occurs in the free flow regime), hence, only a small fraction of the particles gathers on the wall. With the increase of the particle size, the particle inertia increases, which results in their more frequent collisions with the model wall and increasing deposition of drug particles. With the further increasing size (exceeding $25-30 \mu \mathrm{m}$ ), particle impaction with the upper parts of the model wall (mouth and trachea) is very frequent, what drastically reduces the number of particles that could reach the farther structures of the respiratory tract.

The vast majority of the largest particles deposits on the upper part of the trachea and mouth, therefore, just a few particles can reach the bronchi - particle efficacy at the presented segments tends to $0 \%$.

\section{Conclusions}

The presented research indicated that the total deposition of the drug particles on the entire respiratory tract wall is characterized by a sigmoid function that tends asymptotically towards specific values. What is more, when decreasing the particle size and when the specific threshold is reached, the total deposition remains nearly constant even with the further size reduction. Comparing the total deposition results with the data obtained by other research groups, one can observe similar trend functions describing the total deposition dependence on the particle size in the 1-10 $\mu \mathrm{m}$ size range [17], [20], [21], [25]. All the research groups, including ours, indicate that with the increase of the particle size, their inertia and momentum increase as well, which results in a higher impaction with the model wall. As a consequence, a significantly lower number of drug particles can reach farther structures of the bronchial tree and the alveoli. The total deposition values obtained by others are higher than data calculated within the following research, however, those authors did assume different numerical settings [16], [20], [21], [25]. Moreover, the analyzed geometries were reconstructed (or artificially created) for the adult patients, leading to larger flow channels area. Liu et al. [16] used the artificial, idealized and symmetrical models of the children's respiratory tracts and their results of the total deposition were higher as well. Although, they assumed a very high density of the drug (i.e., $1000-3000 \mathrm{~kg} / \mathrm{m}^{3}$ ), which led to a drastic increase in particle inertia and, as a result, higher impaction with the model wall.

Our study introduced the parameter defining the particles that left the numerical domain through the outlets (particle efficacy, $P E$ ). We do think that particles flowing out of the numerical domain will reach farther bronchi generations and the alveoli, increasing the possible drug diffusion to the organism, hence, they should be considered for the analyses. Conducted numerical simulations proved that with the increase of the particle size, their possible efficacy is reduced. However, the particle size decrease tends asymptotically towards specific, constant value. This means that despite inhaling significantly smaller particles, their efficacy might not improve. Unfortunately, one could not find any available references in which the parameter similar to the aforementioned particle efficacy is concerned. Nonetheless, it was decided to compare the results with some general information concerning the dependence of the particle deposition on the particle size. A study of Gizińska et al. [8] suggests that particles of diameters $20-100 \mu \mathrm{m}$ deposit in the nasal cavity, mouth and throat, 6-12 $\mu \mathrm{m}$ particles deposit on trachea and bronchi walls, whereas the particles of $1-5 \mu \mathrm{m}$ in diameter reach the alveoli [8]. Another available referential indicates that particles of diameters exceeding $8 \mu \mathrm{m}$ tend to settle in mouth and throat, 5-8 $\mu \mathrm{m}$ particles deposit in the larger bronchi, whereas particles with diameters of $1-5 \mu \mathrm{m}$ deposit on the farthest bronchi and reach the alveoli [14]. Papers of Gizińska et al. [8], and Kulus and Krenke [14] suggest that the smaller particles $(<1 \mu \mathrm{m})$ are exhaled due to high kinetic energy. Moreover, they indicate that particles which should reach the alveoli and have a therapeutic effect should be in the range of $1-5 \mu \mathrm{m}$. This is consistent with our numerical analyses determining the particle efficacy for pediatric patients.

A lack of further comparable data for pediatric patients suggests a necessity of conducting numerous additional clinical trials as well as further CFD studies. During those researches, the most optimal drug particle size and dose, together with the most optimal shape and ejection parameters of inhalers/nebulizers, 
could be gathered for each patient. As a result, a statistical database for pediatric patients could be created allowing one to perform more personalized and more effective treatment. By and large, it was proven that CFD studies can help in analyzing the flow of drug particles and their distribution along the patient-specific respiratory tract models. This can make it easier to find the right drug dose to get the maximum action in the disease-affected area while minimizing systemic side effects.

\section{References}

[1] Amirav I., Newhouse M.T., Aerosol therapy in infants and toddlers: past, present and future, Expert Rev. Resp. Med., 2008, 2 (5), 597-605,

[2] ANSYS. (2017). ANSYS CFX - theory guide 18.2.

[3] BATCHELOR H.K., MARRIOTT J.F., Formulations for children: problems and solutions, Brit. J. Clin. Pharmaco, 2015, 79 (3), 405-418.

[4] Dalby R., SUMAN J., Inhalation therapy: technological milestones in asthma treatment, Adv. Drug. Deliver. Rev., 2003, 55 (7), 779-791.

[5] Das P., Nof E., Amirav I., Kassinos S.C., Sznitman J., Targeting inhaled aerosol delivery to upper airways in children: Insight from computational fluid dynamics (CFD), PLOS ONE, 2018, 13 (11), e0207711.

[6] FINK J.B., Delivery of inhaled drugs for infants and small children: a commentary on present and future needs, Clin. Ther., 2012, 34 (11), S36-S45.

[7] Fleming S., Thompson M., Stevens R., Heneghan C., Plüddemann A., Maconochie I., Mant D., Normal ranges of heart rate and respiratory rate in children from birth to 18 years of age: a systematic review of observational studies, The Lancet, 2011, 377(9770), 1011-1018.

[8] GizińSKa M., KonARSKa A., RĄGLEWSKA P., RutKowsKi R., StRABURZYŃSKA-LUPA A., Factors affecting efficacy of the aerosol therapy in pediatric patients, Pediatr. Med. Rodz., 2012, 8 (2), 101-106 (in Polish).

[9] Global Initiative for Asthma, Pocket Guide for Asthma Management and Prevention (for Adults and Children Older Than 5 Years): A Pocket Guide for Health Professionals, Global Initiative for Asthma, 2016.

[10] GRISCOM N.T., WOHL M.E., Dimensions of the growing trachea related to age and gender, Am. J. Roentgenol., 1986, 146 (2), 233-237.

[11] Hofmann W., Mathematical model for the postnatal growth of the human lung, Resp. Physiol., 1982, 49 (1), 115-129.

[12] Jodko D., Obidowski D., Reorowicz P., Jóźwik K., Numerical investigations of the unsteady blood flow in the end- -to-side arteriovenous fistula for hemodialysis, Acta Bioeng. Biomech., 2016, 18 (4).

[13] Kennedy M.P., OstrowsKi L.E., Primary ciliary dyskinesia and upper airway diseases, Curr. Allergy Asthm. R., 2006, 6 (6), 513-517.

[14] Kulus M., Krenke K., Children's pulmonology, PZWL Wydawnictwo Lekarskie, Warszawa 2018.

[15] Laube B.L., JansSEns H.M., De JonGH F.H., Devadason S.G., DHAND R., DiOT P., CHRYSTYN H., What the pulmonary specialist should know about the new inhalation therapies, Eur. Respir. J., 2011, 37 (6), 1308-1417.

[16] LiU Z., Li A., Xu X., GaO R., Computational fluid dynamics simulation of airflow patterns and particle deposition characteristics in children upper respiratory tracts, Eng. Appl. Comp. Fluid, 2012, 6 (4), 556-571.

[17] Luo H.Y., LiU Y., Particle deposition in a CT-scanned human lung airway, J. Biomech., 2009, 42 (12), 18691876.

[18] MENTER F.R., Two-equation eddy-viscosity turbulence models for engineering applications, AIAA J., 1994, 32 (8), 1598-1605.

[19] Pedersen S., Dubus J.C., Crompton G., The ADMIT series - issues in inhalation therapy. 5) Inhaler selection in children with asthma, Prim. Care Resp. J., 2010, 19 (3), 209-216.

[20] RAHIMI-GoRJI M., GoRJI T.B., GorJI-BANDPY M., Details of regional particle deposition and airflow structures in a realistic model of human tracheobronchial airways: two-phase flow simulation, Comput. Biol. Med., 2016, 74, 1-17.

[21] Rahimi-Gorji M., Pourmehran O., GorJI-BAndPy M., GORJI T.B., CFD simulation of airflow behavior and particle transport and deposition in different breathing conditions through the realistic model of human airways, J. Mol. Liq., 2015, 209, 121-133.

[22] Reorowicz P., Obidowski D., KŁosiński P., Szubert W., STEFAŃCZYK L., JÓŹWIK K., Numerical simulations of the blood flow in the patient-specific arterial cerebral circle region, J. Biomech., 2014, 47 (7), 1642-1651.

[23] SchueEpP K.G., Devadason S.G., Roller C., Minocchieri S., Moeller A., Hamacher J., Wildhaber J.H., Aerosol delivery of nebulised budesonide in young children with asthma, Resp. Med., 2009, 103 (11), 1738-1745.

[24] Schüepp K.G., Straub D., Möller A., Wildhaber J.H., Deposition of aerosols in infants and children, Journal of Aerosol Medicine, 2004, 17 (2), 153-156.

[25] Tena A.F., ClarÀ P.C., Deposition of inhaled particles in the lungs, Arch. Bronconeumol. (English Edition), 2012, 48 (7), 240-246.

[26] Tyfa Z., Obidowski D., Reorowicz P., StefańczyK L., FORTUNIAK J., JÓŹWIK K., Numerical simulations of the pulsatile blood flow in the different types of arterial fenestrations: Comparable analysis of multiple vascular geometries, Biocybern. Biomed. Eng., 2018, 38 (2), 228-242. 